\title{
DISCUSIONES JURÍDICAS DERIVADAS DE LA LEX TERTIA EN COLOMBIA*
}

César Augusto Castellanos Gómez *

Recibido: Junio 23 de 2015

Aprobado: Noviembre 1 de 2015

\section{RESUMEN}

El presente trabajo tiene como objetivo analizar el fenómeno jurídico de la lex tertia desde el principio de legalidad, mostrando su dinámica en diferentes escenarios de discusión; (i) en el plano jurisprudencial (ii) en el plano de la doctrina del Derecho Penal de producción nacional y foránea; y (iii) desde la teoría del Derecho, con el fin de describir los argumentos que sustentan las posiciones a favor y en contra de la lex tertía y su relación argumentativa con la doctrina del Derecho Penal y la Teoría del derecho en general.

Palabras claves: Derecho Penal, Principio de favorabilidad penal, Lex tertia, Corte Suprema de Justicia de Colombia, Positivismo normativista, Constitucionalismo.

\section{LEGAL DISCUSSIONS DERIVED FROM LEX TERTIA IN COLOMBIA}

\begin{abstract}
This paper aims to analyze the legal phenomenon of lex tertia showing its dynamics in different stages of discussion (i) in the case-law (ii) in terms of doctrine of criminal law of national and foreign sources, and (iii) the law theory, to describe the arguments supporting positions for and against the lex tertia and its argumentative relationship with the doctrine of criminal law and law theory in general.

* El contenido de este artículo es producto del trabajo de grado presentado ante la Universidad Autónoma de Bucaramanga para obtener el título de abogado (2015).

** Abogado de la Universidad Autónoma de Bucaramanga. Especialista en Derecho Comercial. Actualmente cursa el pregrado de Filosofía en la Universidad Industrial de Santander. cesarcastellanosg@gmail.com
\end{abstract}


Keywords: Criminal law, Principle of criminal favorability, Lex tertia, Supreme Court of Colombia, Normative positivism, Constitutionalism

\section{DISCUSSÕES JURÍDICAS ORIGINADAS DA LEX TERTIA NA COLOMBIA}

\section{RESUMO}

Este trabalho tem por objetivo analisar o fenômeno jurídico da lex tertia a partir do princípio da legalidade que mostra a sua dinâmica em diferentes contextos de discussão (i) no nível jurisprudencial (ii) em termos da doutrina do Direito Penal da produção nacional ou forânea e (iii) a partir da teoria do Direito, a fim de descrever os argumentos que sustentam as posições a favor e contra a lex tertia e sua argumentação com a doutrina do Direito Penal e a teoria jurídica em geral.

Palavras-chave: Direito penal, O princípio de favorabilidade criminal, Lex tertia, a Suprema Corte de Justiça da Colômbia, O positivismo normativista, O constitucionalismo.

\section{INTRODUCCIÓN}

El Derecho en la actualidad ya no puede pensarse de forma local. La globalización ha integrado a los países, no solo desde el punto de vista económico sino también desde el punto de vista político y cultural, de las que no ha sido ajeno el Derecho. Las influencias a las que está expuesto el Derecho son muy variadas, desde teóricas que promueven un cambio en la forma de ver e interpretar el Derecho, hasta la creación de normas comunes en cooperación con otros países, pasando por sistemas jurídicos de integración regional e incluso trasplantando fórmulas jurídicas $^{1}$ de éxito de otros continentes ${ }^{2}$.

Así mismo, nuestro sistema jurídico no solo ha recibido influencias exógenas, pues dinámicamente ha permanecido en constante evolución en lo interno a la par que los sistemas políticos intentan a través de

1 Sobre la teoría de los trasplantes jurídicos puede consultarse Bonilla Maldonado, Daniel, Teoría del derecho y los trasplantes jurídicos: Estructura del debate. En: Teoría del derecho y los trasplantes jurídicos. Bogotá. Universidad de los Andes, Siglo del Hombre Editores. 2009.

2 Nuestro sistema penal acusatorio caracterizado por la oralidad en los procedimientos no ha sido una construcción jurídica propia. Estos sistemas procedimentales han sido propios de los países anglosajones y de Estados Unidos y ha sido casi calcado a nuestro sistema por intermedio de la ley 906 de 2004 para agilizar los procedimientos. 
normas jurídicas dar respuesta y adaptarse a la misma velocidad de los cambios sociales y tecnológicos, lo que ha permitido una actualización de normas jurídicas acordes, en el mejor de los casos, a las necesidades del devenir.

Esta evolución endógena ha sido impulsada en gran medida por la investigación jurídica que tiene lugar en los órganos judiciales y en las universidades, principalmente; que han renovado las ideas jurídicas preponderantes en otro tiempo, y han alimentado con percepciones diversas la teoría del Derecho.

Desde esta perspectiva, el Derecho tampoco puede comprometerse de ante mano con una visión dogmática, pues una norma jurídica puede adquirir una connotación diferente aun después de ser creada, según la escuela jurídica e intérprete, lapidando con esto definitivamente la idea de que el sistema puede aún ofrecer respuestas únicas ${ }^{3}$.

Los cambios de normas jurídicas que renuevan drásticamente la forma de ver y actuar en el Derecho, e incluso los cambios de precedente judicial en los órganos colegiados de cierre, son muestras importantes de los cambios exógenos y endógenos del sistema jurídico ${ }^{4}$.

Un ejemplo importante fue la introducción de principios y valores de carácter vinculante en el derecho que trajo el texto constitucional de 1991. Sumado a la creación de la Corte Constitucional se inaugura en Colombia un Derecho principialístico fuerte, donde se modifica de una vez y para siempre la estructura lógica de la norma jurídica, predominantemente cerrada, para dejar de ser exclusivamente una proposición que contiene un supuesto de hecho y una consecuencia jurídica del tipo Si A entonces $B$, por una de textura abierta. Pero las verdaderas implicaciones de este cambio no fueron simplemente lingüísticas sino también un verdadero cambio de paradigma jurídico político, en tanto que, estructuras normativas abstractas solo pueden resolver problemas si se realiza un ejercicio hermenéutico que a fuerza de argumentos indiquen una respuesta correcta, tarea que le corresponde a los jueces y que de contera les da un nuevo papel político que antes los reducía a ser simples vocalistas de la ley.

3 Sobre las diferentes teorías de la interpretación de la norme jurídica puede consultarse: Uprimny, Rodrigo \& Rodríguez, Carlos. Entre deductivismo y activismo: hacía un intento de recapitulación de los grandes modelos teóricos de la interpretación jurídica. [16-12-2014] Obtenido de: http://portalweb.ucatolica.edu.co/ easyWeb2/files/116_10314_deductivismo-activismo.pdf

4 Sobre la vinculatoriedad del precedente jurisprudencial en Colombia puede consultarse: López Medina,

Diego. El derecho de los Jueces. Bogotá. Legis. 2011. 
El Derecho Penal no ha sido ajeno a esta dinámica de cambios. La introducción de la oralidad en el proceso penal, propio de los sistemas penales angloamericanos, fue trasplantada casi en su totalidad, lo que provocó a fuerza de cambio una obligada reestructuración mental del litigante y el juez de la cotidiana labor jurídica.

También la adopción de principios rectores en el Derecho Penal ${ }^{5}$ y del proceso punitivo aportó lo suyo en la codificación de supuestos delictivos, y se estructuraron verdaderas garantías constitucionales y legales a favor de quienes eran investigados o sancionados de cometer algún delito.

No obstante lo anterior, aún el Derecho Penal conserva una estructura predominantemente positiva, y el alcance de los principios aún es objeto de serios debates en los órganos de cierre como la Corte Suprema de Justicia, y el papel político del juez más allá de su labor notarial es todavía un debate no resuelto como veremos en este trabajo.

La intención de estudiar la lex tertia surge al considerar que a partir de la discusión práctica del fenómeno puede interrelacionar la teoría y hacer visible la actualidad de las discusiones que señalamos atrás. Además porque es un fenómeno poco explorado por los investigadores y desde esa perspectiva intelectualmente novedoso.

En síntesis el problema que se aborda en este escrito es porque, tal como se concibe hoy, el principio de favorabilidad penal en Colombia consiste en la posibilidad de acoger, en un contexto de tránsito normativo, la aplicación de la norma más favorable a una situación fáctica particular, no obstante que se ha aceptado mayoritariamente tanto en la doctrina ${ }^{6}$ como en la jurisprudencia que la ejecución de este principio solo es posible si la norma que se acoge es aplicada en su totalidad, es decir, en lo favorable y lo desfavorable.

Ahora bien, ha tomado fuerza en la jurisprudencia la posibilidad de acoger lo más favorable de dos leyes en tránsito normativo y conjugarlas para aplicarlas a un caso concreto (Lex tertia). Empero, esta visión es criticada por vulnerar el principio de legalidad, toda vez que el juez asume un papel creador de normas que no le corresponde y construye una tercera ley que el legislador no ha contemplado. A pesar de todo la jurisprudencia

5 Sobre los actuales fundamentos constitucionales del Derecho Penal puede consultarse: Montealegre Lynett, Eduardo \& Bernal Cuellar, Jaime. El proceso penal, Fundamentos Constitucionales y Teoría General. Tomo I. Bogotá. Editorial Universidad Externado de Colombia. 2013

6 En el capítulo segundo reseñamos la doctrina sobre este punto. 
de la Sala Penal de la H. de la Corte Suprema de Justicia ha aceptado en varios pronunciamientos la posibilidad de la Lex Tertia en Colombia ${ }^{7}$ y esta visión encuentra respaldo en el papel que en el constitucionalismo adquiere el juez como un agente activo en la creación del Derecho, acorde con las necesidades reales, en oposición a la vieja visión pasiva del juez en el positivismo normativista de un aplicador lógico.

Así las cosas, el propósito de este escrito es mostrar cuáles son las discusiones que se derivan de la lex tertia en tres niveles: (i) a partir de los debates que desde el año 2001 se vienen dando en la Corte Suprema de Justicia, (ii) las discusiones que se han dado en la doctrina del Derecho Penal y, (iii) la discusión de la lex tertia vista desde la teoría del derecho en general, específicamente desde el positivismo normativista y el constitucionalismo, con el fin de mostrar la interrelación entre la teoría y la influencia en la práctica judicial colombiana y en sentido contrario, la actualización de la teoría jurídica a partir de nuevos movimientos interpretativos en la dinámica judicial.

Consideramos pertinente este trabajo desde dos dimensiones. En el plano académico tiene pertinencia hermenéutica en la medida en que integra principios a la interpretación y aplicación del Derecho Penal, además plantea un debate doctrinal y jurisprudencial de actualidad relacionado con la aplicación del principio de favorabilidad de la ley penal. En el plano práctico interesa a los jueces, fiscales y estudiosos del Derecho Penal en la medida en que suministra elementos de análisis para determinar si es posible construir una tercera ley sin vulnerar el principio de legalidad.

\section{LEX TERTIA Y CORTE SUPREMA DE JUSTICIA}

En este apartado se propone mostrar qué es la lex tertia a partir de las discusiones que en torno al tema se han generado en la jurisprudencia de la Corte Suprema de Justicia. Con ese propósito el capítulo tiene la siguiente estructura: (i) enunciar cómo se ha tratado el principio de favorabilidad de manera general a partir de la Constitución de 1991 y de forma específica a partir de la ley 599 de 2000, en razón a que es la codificación penal vigente, (ii) mostrar cómo a partir de la interpretación y aplicación del principio de favorabilidad surge la lex tertia, (iii) exponer cómo se ha dado la discusión al interior de la Corte Suprema de

7 Corte Suprema de Justicia, Sala Penal, Sentencia rad. Nº 16.837, del 3 de septiembre de 2001, M. P. Jorge Aníbal Gómez Gallego; Corte Suprema de Justicia, Sala Penal. Sentencia Rad. 19.371 del 26 noviembre de 2003. M.P Álvaro Orlando Pérez Pinzón; Corte Suprema de Justicia, Sala Penal, Sentencia rad. N 19.445, del 6 de octubre de 2004, M. P. Álvaro Orlando Pérez Pinzón; Corte Suprema de Justicia, Sala Penal, Sentencia rad. $N^{\circ} 23.272$, del 27 de agosto de 2007, M. P. Sigifredo Espinosa Pérez. 
Justicia presentando los argumentos más importantes sobre los cuales se han cimentado las posiciones a favor y en contra de la lex tertia.

\subsection{El principio de favorabilidad y la lex tertia}

Para entrar en discusión es menester hacer algunas consideraciones sobre el principio de favorabilidad penal. En Colombia se concibe como una garantía que brinda el Derecho Penal consistente en la posibilidad de acoger, en un contexto de tránsito normativo, la aplicación de la norma más favorable a una situación fáctica particular. Así, este principio guarda estrecha relación con dos principios más, el principio nullum poena sine legem y el principio de derechos adquiridos. Con el primero, en tanto que nadie puede ser castigado por cometer un acto que no se encuentra prohibido y sancionado como delito, lo cual es una garantía propia de los sistemas jurídicos positivistas ya que la acción estatal punitiva está supeditada a la a existencia previa de la ley que la habilite, y debe además ser pública y unívoca. Desde el segundo, los derechos adquiridos están en relación con otro principio que se comporta en su condición de posibilidad, el principio según el cual la ley rige hacia el futuro, y que a pesar de que una ley posterior elimine a la anterior o afecte lo regulado por ella, permanecerá lo reglado hacía el futuro. Ahora bien, este principio se convierte en una verdadera garantía en tanto que un supuesto de hecho delictivo puede ser sometido a una regulación que ya no se encuentre en vigencia o incluso, que se someta a una regulación que no obstante está activa, no lo era al momento de la comisión del acto delictivo.

La Constitución Política indica en el artículo 29 cuál es la funcionalidad de la favorabilidad en los siguientes términos "en materia penal, la ley permisiva o favorable, aun cuando sea posterior, se aplicará de preferencia a la restrictiva o desfavorable" y al respecto la Corte Constitucional en sentencia C-181 de 2002 M.P. Marco Gerardo Monroy Cabra indicó:

Para efectuar la aplicación favorable de la norma y dar entidad al principio mismo se recurre generalmente a dos vías: la de la retroactividad de la ley, fenómeno en virtud del cual la norma nacida con posterioridad a los hechos regula sus consecuencias jurídicas como si hubiese existido en su momento; y la de la ultraactividad de la norma, que actúa cuando la ley favorable es derogada por una más severa, pero la primera proyecta sus efectos con posterioridad a su desaparición respecto de hechos acaecidos durante su vigencia. 
En el mismo sentido la Corte Suprema de Justicia ha resuelto en sus pronunciamientos que:

(...) atendiendo al principio de favorabilidad que rige en materia penal y procesal penal con efectos sustanciales, por disposición del artículo 29 de la Constitución Política y del artículo $6^{\circ}$ de las leyes 600 de 2000 y 906 de 2004, la Sala viene insistiendo en la procedencia de la aplicación retroactiva de la última de las leyes indicadas, a asuntos disciplinados por la primera de las leyes aludidas, por ocurrir los hechos en su vigencia, cimentada en que no solo opera en casos de sucesión de leyes sino, además, en la coexistencia de normas, siempre y cuando los preceptos llamados a regular el asunto jurídico de los dos estatutos procesales contemplen el mismo supuesto de hecho y no hagan parte de la esencia o naturaleza jurídica del sistema procesal penal acusatorio, y el seleccionado le reporte ventajas al procesado o condenado ${ }^{8}$.

Pero en lo que tiene que ver en sus condiciones de posibilidad se ha aceptado mayoritariamente, tanto en la doctrina como en la jurisprudencia, que este principio solo opera si las normas que se encuentran en tránsito regulan supuestos fácticos análogos y en segundo lugar, que la norma acogida por considerarse más benigna en sus efectos ya sea por retroactividad o ultractividad, se acoja en su totalidad, es decir, en lo favorable y lo desfavorable.

Este subprincipio de inescindibilidad de la ley penal se ha trazado durante mucho rato en la Corte suprema de Justicia como el camino que debe seguir el aplicador cuando se encuentre en una situación donde opere el principio de favorabilidad penal, que, dada su fijación jurisprudencial y doctrinal se eleva a criterio hermenéutico ya que su fuente no reside en la ley penal. En otras palabras, según esta línea argumentativa no hay posibilidad de que se pueda tomar lo más favorable de un texto legal y construir con lo más favorable de otro la regla que ajusticia una situación jurídica particular. Pero no siempre ha sido así pues se ha aceptado la Lex tertia, que es la nominación que recibe este fenómeno, que quiere decir tercera ley, y significa la norma que surge a partir de la fusión de lo más favorable de dos normas que regulan un supuesto fáctico análogo y se encuentran en tránsito normativo.

8 Cita de la Corte: Auto 23/03/06, Rad. 18025. Cfr. autos 10/08/05, Rad. 16320 y 04/05/05, Rad. 19094 


\subsection{DISCUSIÓNEN DE LA CORTE SUPREMA DE JUSTICIA}

En la Corte Suprema de Justicia el debate no parece agotado y la posibilidad de que la lex tertia opere no ha tenido respuestas unánimes, pues a pesar de que la tesis mayoritariamente aceptada ha sido que la ley penal ante la favorabilidad no puede escindirse ${ }^{9}$, han existido pronunciamientos en sentido contrario desde el año 2000 con la entrada en vigencia de la ley 599.

En último pronunciamiento sobre el tema la Corte Suprema de Justicia mediante Auto del 24 de febrero de 2014, Rad. 34099, ratifica la línea jurisprudencial que considera inviable la lex tertia que se sustenta sobre la base de dos argumentos que se exponen así:

1. Las funciones políticas no están atribuidas a un juez en el esquema de división de poderes, y por lo tanto la creación de una tercera norma, combinando dos existentes, rebasa la intención del legislador, único legitimado para crear la ley:

El mecanismo de la combinación de leyes que se ha conocido como lex tertia o tercera ley, en la forma en que la doctrina y la jurisprudencia lo vienen concibiendo, abriga una serie de limitantes para el Juez a la hora de aplicar favorablemente disposiciones que se suceden en el tiempo, en la medida en que el devenir de su construcción teórica ha debido solventar los prolegómenos que emergen del esquema tripartita del Poder Público, para no invadirlos, y en ese sentido el fenómeno jurídico no es un acicate de libre.

2. El juez debe garantizar la integridad del ordenamiento jurídico, que se materializa en los principios de unidad, coherencia y plenitud del sistema jurídico; en este sentido no pueden existir dos normas aplicables al caso concreto, y en caso de existir, debe superarse la antinomia y propender por la conservación de la norma "de manera que el celo por la integridad del ordenamiento jurídico, puede decirse que es el faro que guía la conjunción favorable de normas sucedidas en el tiempo".

9 Este argumento fue reiterado en diversos pronunciamientos en las siguientes sentencias. Corte Suprema de Justicia, Sala de Casación Penal, sentencia Rad. 14183 del 31 de enero del año 2002 M.Ps Jorge Córdoba Poveda \& Carlos Augusto Gálvez Argote; Corte Suprema de Justicia, Sala de Casación Penal, sentencia Rad. 21954 del 14 de noviembre del año 2005, M.P Jorge Luis Quintero Milanés; Corte Suprema de Justicia, Sala de Casación Penal, sentencia Rad. 26916 del 4 de noviembre del año 201o, M.P María del Rosario Gonzáles de Lemos; Corte Suprema de Justicia, Sala de Casación Penal, sentencia Rad. 35465 del 24 de agosto del año 2011, M.P María del Rosario Gonzáles de Lemos. 
De los argumentos planteados en esta línea jurisprudencial se puede percibir que, quienes defienden esta tesis se inscriben en la visión jurídica del Derecho Positivo Normativista y su particular teoría del ordenamiento jurídico, donde la norma escrita y producida según los mecanismos regulares por quien está legitimado, adquiere un papel principal en la creación, aplicación y conservación del derecho; bajo el lente de esta postura el juez se limita a administrar las normas legales existentes para resolver los problemas que se le presentan, acudiendo a los principios lógicos, y auxiliado por herramientas como el silogismo, la analogía, o la estructura gramatical de las proposiciones, que se ve plasmada en la jurisprudencia.

En oposición a esta línea jurisprudencial, a partir del año 2000 se inaugura una nueva brecha en la jurisprudencia que hace línea en el sentido de que sí es posible, en nombre del principio de favorabilidad, realizar la conjunción normativa de dos normas que regulan un supuesto fáctico análogo pero que tienen consecuencias diversas. Según esta visión puede desecharse lo desfavorable en el entendido de una verdadera materialidad de la garantía de favorabilidad. Nos referimos a la sentencia del 3 de septiembre de 2001 de la Corte Suprema de Justicia, Sala de Casación Penal, rad. No 16.837, con ponencia del Magistrado Jorge Aníbal Gómez Galleg ${ }^{10}$.

Así, queda trazado el camino de la lex tertia para que mediante otros fallos que se incorporan a la línea formen un precedente jurisprudencial más o menos consolidado. Los fallos más destacados que conforman esta línea son la sentencia del 3 de septiembre de 2001, con ponencia del Magistrado Jorge Aníbal Gómez, rad. № 16.837 (sentencia inauguradora de línea); la sentencia del 26 de noviembre de 2003, con ponencia del Magistrado Álvaro Orlando Pérez Pinzón Rad. 19.371; la sentencia del 6 de octubre de 2004 con ponencia del Magistrado Álvaro Orlando Pérez Pinzón, rad. $\mathrm{N}^{\circ} 19.445$; la sentencia del 21 de marzo de 2007 con ponencia del Magistrado Sigifredo Espinosa Pérez, Rad. 24340; y la sentencia del 27 de agosto de 2007, con ponencia del Magistrado Sigifredo Espinosa Pérez, rad. $\mathrm{N}^{\circ} 23.272$ (ver anexo 1).

10 En ese momento la discusión no fue pacífica pues de nueve magistrados tres aclararon el voto y hubo un salvamento parcial. Las aclaraciones giran en torno a plantar su discrepancia a que se haya aplicado la conjunción normativa, y se justifican en los argumentos atrás reseñados. En cambio, el salvamento de voto parcial planteado por el Magistrado Álvaro Orlando Pérez Pinzón celebró que se haya optado definitivamente por el camino de la lex tertia: "Por supuesto, me identifico con el centro de la decisión tomada y más exactamente con la aceptación que por fin hace la Corte del fenómeno conocido como combinación, conjunción o conjugación de "leyes". En buena hora se reconoce la integridad del principio de favorabilidad". Las razones de su salvamento parcial tuvieron que ver con aspectos diferentes de la sentencia. 
En términos generales, esta línea reposa sobre el argumento de un derecho penal garantista que da prioridad aplicativa al principio que a la regla. En otras palabras, el principio de favorabilidad no puede restringirse de manera tal que implique su anulabilidad, sino que debe propenderse por su materialidad radical, por salvaguardar esta garantía en su integridad. En estos términos se expuso en la sentencia que inaugura esta línea, referenciada atrás:

En primer lugar, la favorabilidad es un problema que ocupa al funcionario judicial en el momento de aplicar la ley, desde luego siempre de cara a una vigencia sucesiva de normas. Es decir, como no es un problema de producción legislativa (legislador) sino de aplicación de la ley (funcionario judicial), debe atenderse al máximo al caso concreto o a la práctica y un poco menos al acervo teórico, con más veras si el propósito legislativo ha sido el de que no se ponga cortapisa a la aplicación de la ley benigna o favorable así definida ("sin excepción", dice el precepto).

En este argumento se percibe una visión jurídica que ya no responde a la que tradicionalmente ha marcado nuestro sistema jurídico, el positivismo, pues la concepción de un derecho que imprima juridicidad a proposiciones normativas que no responden a la estructura lógica de la norma jurídica, como el principio jurídico que se caracteriza por su textura abierta, ha determinado no solo la visión del sistema en su conjunto sino que le ha dado a los jueces un papel activo en la creación del derecho pues son ellos los llamados a especificar el contenido de las normas abiertas mediante la argumentación.

\subsection{DIAGRAMA DEL MOVIMIENTO JURISPRUDENCIAL}

Este movimiento jurisprudencial en la Corte Suprema de Justicia puede graficarse de la siguiente manera, tomando como ejemplo los diagramas de línea del profesor Diego Eduardo López Medina, en el Derecho de los Jueces (López Medina, El Derecho de los Jueces, 2011):

\begin{tabular}{|c|c|c|c|c|c|}
\hline \multicolumn{6}{|l|}{ Tabla 1} \\
\hline \multicolumn{6}{|c|}{ Combinación normativa o lex teria } \\
\hline & 2002 & 2005 & 2010 & 2011 & 2012 \\
\hline $\begin{array}{l}\text { No se } \\
\text { admite }\end{array}$ & \begin{tabular}{|l|} 
Corte Suprema de \\
Justicia, Sala de \\
Casación Penal, \\
sentencia Rad. 14183 \\
del 31 de enero del año \\
2002 M.P Jorge \\
Córdoba Poveda \& \\
Carlos Augusto Gálvez \\
Argote.
\end{tabular} & \begin{tabular}{|l|} 
Corte Suprema de \\
Justicia, Sala de \\
Casación Penal, \\
sentencia Rad. 21954 \\
del 14 de noviembre de \\
año 2005, M.P Jorge \\
Luis Quintero Milanés. \\
\end{tabular} & \begin{tabular}{|l|} 
Corte Suprema de \\
Justicia, Sala de \\
Casación Penal, \\
sentencia Rad. 26916 \\
eldel 4 de noviembre del \\
año 201o, M.P María \\
del Rosario Gonzáles de \\
Lemos.
\end{tabular} & $\begin{array}{l}\text { Corte Suprema de } \\
\text { Justicia, Sala de } \\
\text { Casación Penal, } \\
\text { sentencia Rad. } 35465 \\
\text { del } 24 \text { de agosto del } \\
\text { año 2011, M.P María } \\
\text { del Rosario Gonzáles de } \\
\text { Lemos. }\end{array}$ & $\begin{array}{l}\text { Auto } N^{\circ} \text { AP782-2014 } \\
\text { Rad. (34099). }\end{array}$ \\
\hline
\end{tabular}




\begin{tabular}{|c|c|c|c|c|}
\hline & 2001 & 2003 & 2004 & 2007 \\
\hline Se admite & $\begin{array}{l}\text { Corte Suprema de } \\
\text { Justicia, Sala de } \\
\text { Casación Penal, } \\
\text { Sentencia rad. } \mathrm{N}^{\circ} \\
16.837 \text {, del } 3 \mathrm{de} \\
\text { septiembre de 2001, M } \\
\text { P. Jorge Aníbal Gómez } \\
\text { Gallego }\end{array}$ & $\begin{array}{l}\text { Corte Suprema de } \\
\text { Justicia, Sala de } \\
\text { Casación Penal, } \\
\text { Sentencia rad. } \mathrm{N}^{\circ} \\
19.371 \text {, del } 26 \\
\text { noviembre de } 2003 . \\
\text { M.P Álvaro Orlando } \\
\text { Pérez Pinzón. }\end{array}$ & $\begin{array}{l}\text { Corte Suprema de } \\
\text { Justicia, Sala de } \\
\text { Casación Penal, } \\
\text { Sentencia rad. } \text { N }^{\circ} \\
19.445, \text { del } 6 \text { de } \\
\text { octubre de } 2004, \text { M. P. } \\
\text { Álvaro Orlando Pérez } \\
\text { Pinzón. }\end{array}$ & $\begin{array}{l}\text { Corte Suprema de } \\
\text { Justicia, Sala de } \\
\text { Casación Penal, } \\
\text { Sentencia rad. } \mathrm{N}^{\circ} \\
24340, \text { del } 21 \text { de marzo } \\
\text { de 2007, M. P. } \\
\text { Sigifredo Espinosa } \\
\text { Pérez. }\end{array}$ \\
\hline
\end{tabular}

\section{Elaboración propia}

El panorama regional no es ajeno a estas discusiones pues se ha replicado la visión de la lex tertia en algunos distritos judiciales, que a pesar de la dificultades para acceder a los fallos por adolecer las respectivas relatorías de ciertas fallas en la sistematización de su jurisprudencia, como la publicación de las decisiones por la web y la organización temática, se destacan algunos fallos en donde ha prosperado la línea que acepta la combinación normativa en algunos Magistrados del Tribunal de Bogotá y del Tribunal de Medellín ${ }^{11}$.

Esta dinámica decisional en vez de configurar una línea caótica, en los términos del profesor Diego López Medina, caracterizada por el disenso entre grupos de magistrados, refleja en cambio la coexistencia de dos líneas jurisprudenciales opuestas por la visión ideológica de sus miembros.

Ahora bien, a partir de la Constitución de 1991 se introduce en el derecho colombiano un catálogo de principios y valores que superan la visión del positivismo normativista ${ }^{12}$, pues la obligatoriedad de proposiciones normativas abiertas, incluso por encima de las reglas, cambia por completo la resolución de un problema por medio de un silogismo lógico, ya que la premisa mayor no es precisa por una resolución argumentativa que corresponde al juez. Esto en la medida en que el juez debe propender por la materialización de la justicia y por ofrecer a los ciudadanos verdaderas garantías, al menos en este caso, frente al poder punitivo del Estado, que en ocasiones la regla no puede satisfacer y en cambio el principio jurídico tiene mejores posibilidades.

11 Cfr. Tribunal Superior de Medellín, sala penal, Sentencia del 13 de febrero de 2014 Rad. 2013-3612, M. P. Nelson Saray Botero; y Tribunal Superior de Bogotá, sala penal, sentencia del 11 de febrero de 2014 Rad. 2013-12480-01, M.P José Joaquín Urbano Martínez. Los fallos pueden ser consultados en: http://iustussemillerodeinvestigacion.blogspot.com/2015/06/lex-tertia-sentencias.html

12 Sobre el giro al constitucionalismo en América Latina puede verse López Medina, Diego Eduardo. "Por qué hablar de una Teoría impura del Derecho para América Latina?”, en Teoría del Derecho y los trasplantes jurídicos. Siglo del Hombre Editores. 2009. 
No es coincidencia entonces que el estreno de la línea jurisprudencial que acepta la lex tertia en aras de "mantener la integridad" del principio de favorabilidad haya ocurrido con posterioridad de la vigencia del texto constitucional de 1991 y quizá haya sido por este y por las posturas que frente a los principios, valores y derechos fundamentales ya había dicho la Corte Constitucional colombiana en algunos de sus fallos ${ }^{13}$, que este fenómeno pudo darse y que aún se mantenga.

\subsection{COMENTARIO DE CIERRE}

Se puede cerrar comentando que la combinación de normas o lex tertia es una discusión que apenas se está cimentando y al parecer, la dinámica se alimenta según la formación ideológica de los jueces y magistrados que sucedan los escenarios colegiados y judiciales. Y más allá de la lex tertia, la discusión planteada de fondo versa sobre la teoría jurídica: ¿De qué manera influye en las decisiones judiciales la visión que del derecho tenga un juez o un magistrado? ¿Cuál es su papel real en la construcción jurídica? ¿Hasta qué punto las diferentes visiones fomentan la actualización del Derecho? De manera que hasta este punto, si bien se ha logrado identificar las líneas jurisprudenciales al interior de la Corte Suprema de Justicia, se abren otras discusiones derivadas de la lex tertia que desbordan la intención de este texto, pero que invitan a ser exploradas posteriormente.

\section{LEX TERTIA Y DOCTRINA DEL DERECHO PENAL}

En este apartado se propone reseñar algunos autores destacados de la doctrina nacional e internacional, que nos permitan hacer una idea sobre la evolución histórica y el estado actual de la discusión en torno a la conjunción normativa o lex tertia desde la dogmática penal. Para ello señalaremos dos momentos en la discusión que se componen de lo siguiente: En el primer momento se reseñará la evolución histórica de las escuelas del derecho penal y su relación o aporte a la discusión de la lex tertia. En un segundo momento se reseñarán libros, artículos y ensayos de investigación, de autores nacionales e internacionales, que mediante los trabajos han realizado los aportes a la discusión, pero que se distinguen del primer momento porque no están inscritos de forma abierta en ninguna de las escuelas penales.

$13 \mathrm{Al}$ respecto puede consultarse las sentencias de la Corte Constitucional, C-479 de 1992 M.P José Gregorio Hernández Galindo y Alejandro Martínez Caballero; Sentencia C- 592 de 2005 M.P Álvaro Tafur Gálvis; Sentencia C-371 de 2011, M.P. Luis Ernesto Vargas Silva; Sentencia de T-567 de 1998 M.P. Eduardo Cifuentes Muñoz, entre otras. 


\subsection{Primer momento de la Lex tertia en la doctrina de Derecho Penal}

Para cumplir con el propósito señalado es indispensable definir los términos sobre los cuales recorreremos la evolución histórica de la discusión en torno a la conjunción normativa desde la escuelas del Derecho Penal, y en ese sentido, se entiende por escuela aquella visión del derecho penal que aglutina a diferentes teóricos de acuerdo a sus posturas que sobre conceptos como delito, legitimidad de la sanción, sobre el fin de la pena y en general sobre conceptos básicos comparten, y que se diferencian de otras visiones jurídicas.

Desde esta perspectiva creemos justificado presentar un recorrido por las escuelas más importantes del Derecho Penal para caracterizarlas e identificar la aparición del principio de favorabilidad y el fenómeno de la LexTertia.

\section{Escuela Clásica del Derecho Penal.}

Entre sus principales exponentes se encuentran Enrico Ferri (18561829), Cesare Beccaría (1738-1794), Francesco Carrara (1805-1848) y Gaetano Filangieri (1752-1758), entre otros, y sus postulados precisan un Derecho Penal contenido en normas de Derecho positivo dictadas por el legislador (Beccaria, 2010), no obstante direccionadas bajo los parámetros de un Derecho natural que trazara su finalidad y que además era su fuente, frente al cual no podía haber contradicción.

Consecuente con ello, se aceptaron algunos principios como "nullum pena sine lege" y "nullum crime sine lege" en el sentido en que el Estado tendría una potestad sancionadora pero reglada, la cual posibilitaría de alguna manera predecir las consecuencias de los actos y los procedimientos por los cuales operaria (Beccaria, 2010).

En cuanto a la sanción penal se consideró que la pena es un mal que infringe el Estado para restablecer el orden jurídico, que se impone de acuerdo con las formalidades legales. La responsabilidad penal era observada de acuerdo a una valoración triple que debía realizar el aplicador de justicia, en primer lugar una imputación física consistente en establecer la causa material del hecho punible, una imputación moral que se reduce a establecer la libertad y consciencia de realización del hecho y en tercer lugar una imputación legal, es decir la verificación de prohibición legal del hecho (Velásquez, 2009). 
Sin embargo el proceso penal era en aquel tiempo muy rudimentario, y apenas afloraban las garantías penales, por lo que se puede subrayar entre los aportes más importantes a la discusión el hecho de que empezaran a constituirse derechos y principios rectores en los procesos que a la postre fue un trazo crudo de verdaderas garantías contra el poder punitivo del Estado. El principio de legalidad no tardó en dar paso a la doctrina de los derechos adquiridos, y con el tiempo al principio de favorabilidad.

\section{Escuela Positivista del Derecho Penal.}

La Escuela Positivista, influida por los nuevos paradigmas políticos que trajo la revolución francesa, a la par del desarrollo de las ciencias naturales y atraída por la novedad del método científico y su aporte a la ampliación de las fronteras del conocimiento (Velásquez, 2009), quiso hacer del Derecho Penal una ciencia que partía del análisis del delito describiéndolo en sus elementos constitutivos sin acudir a elementos externos de la construcción normativa, echando mano de la lógica y del análisis gramatical para construir su dogmática.

Dentro sus principales exponentes, sin ser los únicos, se encuentran Franz Von Lizt, Karl Biding (1841-1920) y Ernst Von Beling (18661932), retomando los avances de la Escuela Clásica como la necesidad de claridad y completud de las normas penales (Velásquez, 2009), y reformulados los conceptos ya bajo una nueva visión del Estado y del poder público, como el principio de legalidad que tenía su importancia para definir competencias tanto de quién hace la ley hasta quién la ejecuta pero también de cuáles son los delitos y cuáles son los procedimientos para aplicar a la ley.

También la visión humanista de la Ilustración tuvo efecto en esta escuela pues la legalidad no era vista solo en su utilidad de principio que fortalece la visión del Derecho como ciencia sino también, teniendo en cuenta la importancia política del Derecho Penal, era vista como un verdadero control a la potestad de castigar del Estado, que redundaba en garantía para los gobernados.

En esta escuela, al igual que en la Clásica, es notoria su complementariedad con la teoría del Positivismo Normativo del que hablaremos más adelante, y esclarece los antecedentes de los argumentos que sustenta la tesis según la cual no es posible darse la conjunción normativa, pues en esta visión se requiere una norma 
habilitadora para construir el derecho, que por regla general corresponde al legislador y en ningún caso al juez.

\section{Escuela Finalista delDerecho Penal.}

Como figura más representativa de esta escuela encontramos a Hans Welzel (1904-1977) jurista alemán que vivió la Segunda Guerra Mundial y que según sus críticos (Velasquez, 2009) fue fundamental para la formulación de los postulados de la Escuela Finalista. Entre los más importantes destacamos que él considera que el Derecho, concebido en el sentido de la escuela Positivista, mostraba claramente su debilidad para sustentar la teoría penal pues se distanciaba de la realidad y se convertía en un instrumento de poder al servicio del terror estatal, por eso el Derecho debía buscar por fuera de este un postulado principalístico, un catálogo axiológico, unos principios inmanentes y universales que no pudieran ser desconocidos tranzando una finalidad al Derecho y a la vez sean su vigía.

Este catálogo de principios se acompasa a las democracias constitucionalistas nacientes en Europa, y al reconocimiento de principios y derechos fundamentales recogidos en textos primarios o normas de reconocimiento, que a la postre modificaron sustancialmente los conceptos de la teoría del delito y del proceso penal.

En consecuencia, esta escuela está a disposición de armonizar con los principios, valores y derechos que se reconozcan o se identifiquen, dando lugar a un Derecho Penal complejo que obliga a un estudio sistemático para su comprensión en la medida que no se reduce simplemente al compendio de normas penales sino que va más allá, incluso a elementos meta-jurídicos o por fuera del derecho.

Y desde allá, donde se empieza a hablar de principios que rigen la actividad punitiva del Estado, se estructuran principios como el de proporcionalidad de la sanción penal y el principio de favorabilidad penal. No obstante su novedad, no se plantearon la posibilidad de la combinación de normas en aplicación de la favorabilidad, pero la constitución del principio es un gran avance en ese propósito. Nótese que desde esa escuela ya se venía cuestionando la teoría jurídica positiva en tanto que se erigía como límite, o quizá como un obstáculo, en la actualización del Derecho Penal a la par de los avances en el Derecho Político y Constitucional. 


\section{Escuela Funcionalista del Derecho Penal.}

A diferencia de las anteriores escuelas donde el juez cumple un papel de vocero de la ley, un simple operario lógico de la justicia, en la escuela Funcionalista adquiere un papel muy importante, ya que, para el estudio de casos concretos se deben considerar otras variables que escapan a la mera nomología, como por ejemplo, la política criminal de un Estado, el desarrollo de otras ciencias humanas y su aporte al conocimiento del hombre como la psicología, la psiquiatría, la sociología y finalmente, el papel enriquecedor que para el Derecho ofreció la criminología (Roxin, 2003).

Esta escuela acompasa con un Derecho Constitucional que ofrezca un catálogo de principios garantes del buen proceso, es más, para esta escuela la racionalidad de la pena supone la existencia de garantías reales que determinen las coordenadas de la función castigadora del Estado, y el principio de favorabilidad adquiere un importancia no antes vista, al punto de que es a nivel de los postulados de esta escuela en donde es posible plantear la posibilidad de la conjunción normativa sin que riña teóricamente con el cuerpo dogmático.

Entre los juristas más destacados de esta escuela se puede incluir al profesor Claus Roxin, quizá el jurista más influyente en la construcción dogmática penal colombiana actual; no obstante, en su libro Derecho Penal General aborda solo de forma orbital la conjunción normativa, pues no se refiere directamente al fenómeno ni lo identifica con el enunciado latino, solo se limita a enunciar que en la aplicación del principio de favorabilidad la norma escogida debe "ser aplicada como un todo" y por tanto no hay lugar a un punto intermedio. Esta argumentación concuerda con la visión positivista tradicional, en tanto que la norma no puede combinarse por ir en contra de la legalidad y darle atribuciones legislativas que son eminentemente políticas, a quien desde su concepción le han sido limitadas: el juez.

Compartiendo la misma postura del profesor Roxin pueden identificarse en la doctrina nacional al profesor Hernando Velásquez Velásquez con su voluminosa obra titulada Derecho Penal, Parte General, que de igual manera cierra la discusión sobre la conjunción normativa en tanto que considera que de la aplicación del principio de favorabilidad de la ley penal, se desprende el subprincipio de inescindibilidad de la ley, por lo tanto debe aplicarse la ley que resulte más favorable como un todo, es decir, también con lo perjudicial. 


\section{.2 Segundo momento de la Lex tertia en la Doctrina del Derecho Penal}

Como un segundo momento, en donde ya hay nuevas visiones jurídicas en la palestra, se puede mencionar entre los juristas, profesores e investigadores que ya conocen el fenómeno jurídico de la conjunción normativa, independientemente de si están o no de acuerdo con esa postura, y en el ámbito internacional, al profesor Raúl Baldomino Díaz quien es el autor del artículo de investigación titulado "Retroactividad de las modificaciones a la norma complementaria de una ley penal" (Baldomino, 2009) donde de forma indirecta estudia el fenómeno jurídico de la lex tertia dejando evidencia de que existen dos posiciones contrapuestas en la doctrina jurídica, una posición mayoritaria y acogida por el autor considera que tomar lo más favorable de dos leyes penales vigentes y aplicarla a un caso concreto vulnera la legalidad (lege sripta) en tanto que se construiría una tercera ley que no les corresponde a los jueces en sus funciones. La segunda posición es reflejada en dos jurisprudencias de la Corte Chilena de cierre en lo penal y referenciadas por el autor, que han aceptado la lex tertia.

En el ámbito nacional se puede encontrar el libro Estudios de Derecho Penal (Kawaguchi \& Suárez López, 2010) que hace una recopilación de ensayos y artículos sobre diferentes temáticas del Derecho Penal, publicado por la Universidad Jorge Tadeo Lozano. Se resalta el texto titulado el "Principio de favorabilidad: algunas problemáticas que cuestionan su concepción tradicional" escrito por el profesor Carlos Alberto Suárez López; en donde se propone hacer evidentes algunas nuevas concepciones surgidas en la doctrina penal que han puesto en cuestión la concepción habitual del principio de favorabilidad en la ley y la jurisprudencia. Entre ellas la problemática reseñada en el acápite tercero de su artículo estudia el fenómeno de la lex tertia y lo titula "Creación de una tercera ley o lex tertia por vía del principio de favorabilidad”, en donde tiene como propósito señalar en primer lugar a qué se le denomina lex tertia o tercera ley, cuándo estamos frente a un fenómeno de esta naturaleza, y resalta el debate que ha surgido al interior de la Sala Penal de la Corte Suprema de Justicia que en ocasiones ha aceptado la configuración de la lex tertia. Empero el autor toma posición en la discusión al señalar que no comparte la aplicación de la lex tertia en Colombia por los mismos argumentos ya en otras veces repetidos.

El profesor y otrora fiscal seccional de Bogotá Antonio Luis Gonzáles Navarro en su ensayo titulado "El principio constitucional de 
favorabilidad penal" (Gonzáles Navarro, 2014) señala que en Colombia se ha presentado el fenómeno de la lex tertia o también conocida como conjunción normativa, e indica que es un fenómeno que puede seguir dándose en la jurisprudencia de la Corte Suprema de Justicia, no obstante enuncia el tema de contera sin profundizar en él.

Finalmente, como la más reciente publicación de doctrina nacional puede señalarse el texto publicado por los profesores Eduardo Montealegre Lynett y Jaime Bernal Cuéllar, titulado El Proceso Penal, Tomo II, Estructura y garantías procesales, en cuyo texto reseñan que la Corte Suprema de Justicia ha aceptado la tesis de la combinación normativa y presentan el argumento expuesto en la sentencia de la Sala Penal de la Corte Suprema de Justicia del 3 de septiembre de 2001, con ponencia del Magistrado Jorge Aníbal Gómez rad. No 16.837.

\subsection{Comentario de cierre}

A diferencia de la jurisprudencia de la Corte Suprema de Justicia, entre las doctrinas reseñadas se puede hacer los siguientes comentarios: (i) de la doctrina reseñada en el primer momento, apenas se está construyendo al interior de la dogmática penal el lenguaje de principios como garantías a los ciudadanos hasta la estructuración, por ejemplo, del principio de favorabilidad; sin embargo, parece que la lex tertia es un fenómeno desapercibido o poco conocido por los autores reseñados, o quizá no se concebía siquiera la posibilidad de que se diera la conjunción normativa. De otra parte, es el Funcionalismo, y quizá el Finalismo, las visiones dogmáticas en las cuales puede darse la discusión de la conjunción normativa o lex tertia, debido al lenguaje axiológico del que se abastecen estas estructuras conceptuales; (ii) del segundo momento, cuando la lex tertia ya es un fenómeno conocido, este no ha ocupado una atención central entre los investigadores y juristas, esto explica la reducida discusión en el tema y los escasos textos que han abordado el problema; (iii) por otra parte, en este segundo momento parece mayoritariamente de acuerdo en que no es posible la lex tertia derivada del principio de favorabilidad, compartiendo los argumentos de la jurisprudencia de la Corte Suprema de Justicia, o toman una actitud más objetiva reseñando solo cuál es la problemática que se discute y advirtiendo que el fenómeno se ha presentado en alguna sentencias.

\section{Teorías del Derecho como condición de posibilidad de la Lex tertia.}

En este apartado se propone referenciar diferentes teorías normativas con sus postulados más importantes con el fin de exponer en qué medida 
éstas riñen con la lex tertia, o mejor, en cuál de éstas hay más condiciones de posibilidad del fenómeno en estudio. Esto se explica en tanto que de esta manera también podemos justificar en qué medida la teoría puede influir sobre la práctica judicial y en cierta forma, la determina. Con este propósito este capítulo tendrá dos componentes: en primer lugar se reseñará el Positivismo Normativista como teoría jurídica que se ubica a un lado de la balanza donde no es posible la combinación normativa $\mathrm{y}$, en segundo lugar, reseñaremos el Constitucionalismo, que se ubica en el lado opuesto donde puede o no darse el fenómeno de la conjunción normativa.

\subsection{El Positivismo normativista y la lex tertia.}

Hablar de Filosofía del Derecho en estricto sentido no fue posible sino hasta finales del siglo XIX a partir de los trabajos de Rudolf Stamler, seguido por otros pensadores como Kelsen, Gustav Radbruch, entre otros (Siches, 1974). Un ejemplo de esto fue cómo teóricos del derecho se aproximaron desde el Positivismo Normativo a abordar verdaderos problemas filosóficos: cuestiones en torno al conocimiento del Derecho y a su deber ser fueron inaugurales de esta filosofía.

Particularmente, Hans Kelsen ofrece al Derecho toda una justificación filosófica que lo desmarca de otros saberes y formas de conocimiento para acercarlo a una visión de ciencia. Le da estructura lógica completa, una visión totalizadora que se crea a sí mismo y se justifica. Racionaliza el fenómeno jurídico y lo soporta en conceptos como unidad, coherencia y plenitud; de manera tal que se llega a creer que el sistema puede ofrecer respuestas únicas y ciertas a los problemas jurídicos ${ }^{14}$, bajo la utilización de los principios de la lógica formal y el silogismo deductivo.

El Positivismo Normativista, como se dijo, concibe al Derecho como un gran sistema de normas (Bobbio, 2007) que se estructuran a la luz de tres conceptos que conforman su teoría del ordenamiento, primacía de la norma escrita, unidad, coherencia y plenitud del sistema. Se entiende desde el concepto de unidad que la norma jurídica escrita hace parte del ordenamiento, y por tanto es obligatoria, solo cuando ha sido expedida por quien tiene competencia para ello, y este a su vez legitima su competencia en otra norma de categoría superior, y así sucesivamente

14 La pretensión de respuestas únicas es explicada por el éxito que en las ciencias naturales logró el método científico que partiendo del análisis de fenómenos se descubrían reglas únicas y verdaderas. La positivización de las ciencias naturales se irrigó a las ciencias sociales que vieron en su modelo metodológico la posibilidad de hallar reglas verdaderas y de esta manera convertirse en ciencia, por lo cual se positivaron no solo el derecho sino también la psicología, la sociología, la historia (Cassirer, 2008). 
hasta llegar a la norma fundamental, formando un solo cuerpo normativo.

Por el concepto de coherencia, la norma jurídica se entiende parte de un orden y por tanto, en relación armónica con el todo y con las demás partes constitutivas. Esta visión de orden y armonía es necesaria para no incurrir en contradicciones lógicas, denominadas antinomias. Sin embargo, la existencia de normas incompatibles entre sí hace que el sistema dote al aplicador del Derecho con diversos criterios para superarlas. Así se han señalado criterios lógicos que apuntan a diferencias cronológicas, jerárquicas y de especialidad de las normas para identificar cuál es la aplicable a un caso concreto ${ }^{15}$.

El concepto de plenitud predica del ordenamiento jurídico su cualidad de regular todos los casos concretos mediante una norma. Así se parte de un criterio de suficiencia y de razón a priori según el cual el legislador puede anticipar en su regulación el comportamiento humano mediante normas jurídicas en todos los aspectos, y especialmente en aquellos actos que despliegue un individuo en relación con los otros.

La norma jurídica mantiene una estructura lógica predeterminada por un supuesto de hecho y una consecuencia, que debe estar en consonancia con el sistema jurídico por su claridad, por su completud, es decir por ser suficiente en sí misma para resolver el problema para la cual fue prevista.

Como se observa, en esta teoría el juez está restringido totalmente por el ordenamiento jurídico, su papel es el de un mero vocalizador de la ley, previo proceso de identificación de la norma aplicable al caso concreto. El sistema jurídico establece las premisas mayores y universales y la realidad fáctica la premisa menor para perfeccionar el silogismo deductivo de la lógica formal.

Se descarta así la creación judicial de Derecho, pues se parte del presupuesto de que el Derecho es pleno, univoco, y en perfecta unidad; por tanto, todas las respuestas a los problemas jurídicos se hallan en él, incluso, si no existiera la norma aplicable al caso concreto, y se inventara una norma jurídica o se combinaran diferentes normas para crear la solución, ésta no gozaría de validez como premisa mayor, pues el juez adolece de la competencia para crear normas que otorga otra norma

15 Sobre estos criterios consúltese Norberto Bobbio, Teoría general del Derecho, publicada por Editorial Temis o Marco Gerardo Monroy Cabra, Introducción al Derecho, publicado por Editorial Temis. 
jurídica habilitadora $^{16}$, y que por regla general le ha sido asignada al legislador.

Así que una teoría afianzada en la lógica no da cabida a la razonabilidad del juez o la reduce bastante, pues la norma jurídica caracterizada por su claridad, su univocidad, y su completud, relegaría al juez al rol de adaptador del supuesto fáctico a la hipótesis legal para configurar el silogismo jurídico perfecto. Tampoco habría posibilidad de concebir normas de textura abierta, como los principios jurídicos, en tanto que la claridad se perdería, y sobre todo, se descartaría en esta visión cualquier conjunción normativa o lex tertia pues se rompería la integridad del sistema jurídico al no hacer uso de las estrategias lógicas del sistema para superar la antinomia que supone dos normas regulando un mismo supuesto fáctico. En términos generales se estaría lesionando el principio de legalidad.

\subsection{El Constitucionalismo y la lex tertia.}

El Constitucionalismo se ha erigido en su acepción jurídica (Comanducci, 2011) como un modelo según el cual la validez de una norma esté condicionada por su armonía con el texto constitucional, armonía que dicho sea de paso no se reduce solo a aspectos procedimentales, sino también en cuanto a su contenido. El texto constitucional se caracteriza por su superioridad frente a otras normas del sistema jurídico, y en lo sustancial, porque organiza el Derecho Político de un Estado, (denominado Constitucional de Derecho) y establece las garantías mínimas de los ciudadanos (derechos fundamentales).

Ahora bien, en el Constitucionalismo ${ }^{17}$ la estructura de la norma se replantea con la estructura gramatical de las proposiciones que

16 Herbert Leonard Hart plantea que en un ordenamiento jurídico existen dos tipos de reglas. En primer lugar se pueden distinguir las reglas primarias que se identifican porque en su estructura lógica formulan una obligación y su forma gramatical se compone de elementos facticos y de una consecuencia lógica, es decir, un modelo tradicional de norma jurídica que regularía un caso concreto y que establece una sanción. En segundo lugar, existen normas dentro del ordenamiento jurídico que hacen posible que las normas primarias se distingan de cualquier otro orden normativo distinto al jurídico, y en el que están en constante relación de pertenencia o determinación. A estas las denomina el autor como reglas secundarias o de segundo orden, que a su vez se clasifican en (i) la regla de reconocimiento, (ii) reglas de cambio y (iii) reglas de adjudicación. Nos interesa la regla de reconocimiento pues abre una pequeñísima brecha al activismo judicial pues en ausencia de una norma particular para resolver un caso difícil, la norma de reconocimiento habilitaría al juez para que resuelva la situación, brecha que queda en la discrecionalidad del juez, que en todo caso sigue siendo relativa pues su decisión debe estar en armonía con el sistema y en todo caso habilitado por la norma de reconocimiento.

17 Puede consultarse el ensayo de Luigi Ferrajoli, Constitucionalismo principalista y Constitucionalismo garantísta, publicado por la revista Doxa 34 (2011). 
reconocen derechos, en tanto que ya se supera el prejuicio de la norma escrita que satisfaga todos y cada uno de los supuestos facticos posibles, sino que una redacción abierta basta para ofrecer protección jurídica. Además, contienen un catálogo de principios y valores vinculantes que determinan las coordenadas de la acción Estatal (ya sea en el ámbito político-administrativo o judicial). Asimismo, se erigen Cortes de cierre en lo constitucional, que tienen como finalidad interpretar los preceptos del texto constitucional, atribuyéndoles un papel activo en la materialización de principios y derechos de los ciudadanos, dándole especificidad y sentido mediante la argumentación.

Colombia, si bien ha tenido una historia constitucional relativamente volátil (Quinche Ramírez, 2010), estrena un "constitucionalismo actual” a partir de la promulgación de la Constitución Política en 1991, con influencia de los textos constitucionales de Italia (1948) y de España (1978), y que cambia de forma definitiva el panorama jurídico. El catálogo axiológico que contiene la Constitución tuvo sus efectos inmediatos pues condicionaba la exequibilidad de una norma o su interpretación, fenómeno que empezó a denominarse como la "constitucionalización del Derecho".

El Derecho Penal colombiano no quedó atrás pues los principios y derechos fundamentales han contribuido a su actualización, a manera de ejemplo señalamos el artículo 29 que eleva la favorabilidad penal a rango de principio constitucional y derecho fundamental, y que derivada de su aplicación preferente y radical ha dado nacimiento al fenómeno de la conjunción normativa o lex tertia.

Como quiera que los enunciados constitucionales, como se dijo, poseen una textura abierta, corresponde a los jueces determinar a fuerza de argumentos cual es el alcance de dicho principio y derecho, lo que en últimas ha dado lugar a un derecho argumentativo, de preferencia persuasiva y convencimiento del auditorio.

No obstante este activismo judicial no es del todo arbitrario ni discrecional pues su interpretación debe estar en consonancia con los demás principios y normas constitucionales.

Creemos entonces que las posturas jurisprudenciales que permiten la conjunción normativa, comparten una visión jurídica en esta dirección, siendo el Constitucionalismo, con su lenguaje de principios y derechos, en donde se dan las mejores condiciones de posibilidad. 


\subsection{Comentario de cierre.}

Todo indica que solo sobre la base teórica del Constitucionalismo, en cuya estructura de principios y garantías está soportado el sistema jurídico, puede argumentarse la posibilidad de la conjunción normativa o lex tertia. Esto contribuye a entender la postura teórica o la visión del derecho que comparten, por ejemplo, los grupos de magistrados que argumentan a favor y en contra del fenómeno en estudio.

\section{CONCLUSIONES}

Proponer el estudio de un fenómeno jurídico desde diferentes ópticas (jurisprudencial, dogmática y teórica) debe tener como resultado la comprensión como pretensiones totalizadoras del fenómeno. En este caso, de quiso explorar este camino solo a partir de las discusiones que en torno al fenómeno se han generado, y en esa medida, quedan abiertas otras posibilidades de análisis.

Ahora bien, la lex tertia es un fenómeno jurídico novedoso que se encuentra en discusión, como se vio, en diferentes grados de intensidad. Los avances que se logren en alguna de las tres discusiones tendrán consecuencias naturales sobre las demás. Como se ha tratado de hacer visible, la teoría puede determinar la práctica judicial, y esta, puede promover nuevos desarrollos teóricos y dogmáticos. Ese ha sido el caso de la lex tertia, fenómeno que se ha dado como consecuencia de la introducción en el derecho de catálogos axiológicos, que poco a poco se han desligado al sistema, de la tradicional concepción positivista y su teoría del ordenamiento, o que la han actualizado, y superando la visión de un Derecho unívoco y completo. El suplantamiento teórico lo ha dado el constitucionalismo, que ha permeado en todo el Derecho, reexplicándolo. A nivel dogmático la Escuela Funcionalista brega en menor medida por su constitucionalización pues se ha familiarizado con la existencia de principios y valores jurídicos. No obstante, el cambio de cuerpos normativos es un proceso lento y gradual, por lo que en el entre tanto deben reinterpretarse los existentes, lo que ha dado lugar a estas discusiones como la lextertia.

A pesar de las actualizaciones que el siglo $\mathrm{XX}$ trajo a la visión del Derecho Positivo Normativista, como el lenguaje, principios, o el reconocimiento de derechos fundamentales, y de otras visiones diferentes a ésta como el constitucionalismo que dicho sea de paso sostiene el profesor Diego López (López Medina, 2009) que el Derecho 
Colombiano se ha nutrido tardíamente, el Derecho Penal sigue estructurado en ella. La norma penal, por la implicaciones políticas y su importancia para el mantenimiento de la paz general conserva entre sus características 1) un supuesto fáctico que prohíbe, y que no puede contener ambigüedades en su redacción, y una consecuencia jurídica o sanción clara que se simplifica en la fórmula Si A entonces B 2) su estructura da una margen de discrecionalidad judicial mínimo, pues de alguna manera se puede anticipar la consecuencia con solo identificar la hipótesis jurídica. Ello explica también por qué la renuencia de muchos investigadores y juristas en aceptar la conjunción normativa, pues iría contra un principio lógico de suprema importancia para la interpretación, el tercero excluido.

\section{REFERENCIAS BIBLIOGRÁFICAS}

Beccaria, C. (2010). De los delitos y de las penas (3ra ed.). Bogotá: Editorial Temis.

Bernal Cuéllar, J., \& Montealegre Lynett, E. (2013). El Proceso Penal, Estructura y garantías penales (Vol. II). Bogotá: Universidad Externado de Colombia.

Bernal Cuéllar, J., \& Montealegre Lynett, E. (2013). El Proceso Penal, fundamentos constitucionales y teoría general (Vol. I). Bogotá: Universidad Externado de Colombia.

Bobbio, N. (2007). Teoría general del Derecho. Bogotá: Editorial Temis.

Bonilla, D. (2009). Teoría del Dercho y los trasplantes jurídico: Estructura del debate. En D.

Bonilla, D. López, D., et al. (2009) Teoría del derecho y trasplantes jurídicos, p.11-36. Bogotá: Siglo del Hombre Editores.

Cabra, M. . (2003). Introducción al Derecho. Bogotá: Temis S.A.

Cassirer, E. (2008). Filosofía de la Ilustración. Mexico D.F: Fondo de Cultura Económica.

Comanducci, P. (2011). "Constitucionalismo": problemas de definición y tipologías. Doxa(34), $95-101$. 
Colombia. Corte Constitucional. (12 de marzo de 2002). Sentencia C181. M.P. Marco Gerardo Monroy Cabra.

Colombia. Corte Constitucional. (13 de Agosto de 1992). Sentencia C479. M.P José Gregorio Hernández Galindo y Alejandro Martínez Caballero.

Colombia. Corte Constitucional. (9 de junio de 2005). Sentencia C592. M.P Álvaro Tafur Galvis.

Colombia. Corte Constitucional. (11 de mayo de 2011). Sentencia C371. M.P. Luis Ernesto Vargas Silva.

Colombia. Corte Constitucional. (7 de octubre de 1998). Sentencia T567. M.P. Eduardo Cifuentes Muñoz.

Colombia. Corte Suprema de Justicia. Sala de Casación Penal. (3 de setiembre de 2001). Rad. Nº 16.837. M. P. Jorge Aníbal Gómez Gallego.

Colombia. Corte Suprema de Justicia. Sala de Casación Penal. (26 noviembre de 2003). Rad. N ${ }^{\circ}$ 19.371. M.P Álvaro Orlando Pérez Pinzón.

Colombia. Corte Suprema de Justicia, Sala de Casación Penal. (6 de octubre de 2004). Rad. N 19.445. M. P. Álvaro Orlando Pérez Pinzón.

Colombia. Corte Suprema de Justicia. Sala de Casación Penal. (14 de noviembre del año 2005). Rad. $N^{\circ}$ 21954. M.P. Jorge Luis Quintero Milanés.

Colombia. Corte Suprema de Justicia. Sala de Casación Penal. (21 de marzo de 2007). Rad. N²4340. M. P. Sigifredo Espinosa Pérez.

Colombia. Corte Suprema de Justicia. Sala de Casación Penal. (27 de agosto de 2007). Rad. N²3.272.M. P. Sigifredo Espinosa Pérez.

Colombia. Corte Suprema de Justicia. Sala de Casación Penal. (20 de enero de 2010). Rad. N²6692. M. P. Alfredo Gómez Quintero.

Colombia. Corte Suprema de Justicia. Sala de Casación Penal (4 de noviembre del año 2010). Rad. $N^{\circ}$ 26916. M.P María del Rosario Gonzáles de Lemos. 
Colombia. Corte Suprema de Justicia. Sala de Casación Penal. (24 de agosto del año 2011). Rad. N 35465. M.P María del Rosario Gonzáles de Lemos.

Colombia. Corte Suprema de Justicia, Sala de Casación Penal. (24 de febrero de 2014). AP782-2014 rad. N 34099. M.P Fernando Alberto Castro Caballero.

Colombia. Tribunal Superior de Medellín. Sala Penal. (13 de febrero de 2014). Rad. N²013-3612. M. P. Nelson Saray Botero.

Colombia. Tribunal Superior de Bogotá. Sala Penal. (11 de febrero de 2014). Rad. Nº.2013-12480. M.P José Joaquín Urbano Martínez.

Ferrajoli, L. (2011). Constitucionalismo principalista y Constitucionalismo garantista. $\operatorname{Doxa}(34), 15-54$.

Gonzales, A. L. El principio constitucional de la favorabilidad penal. $\mathrm{R}$ e c u p e r a d o https://www.google.com.co/url? sa=t\&rct=j\&q=\&esrc=s $\&$ source $=$ web $\& \mathrm{~cd}=10 \& \mathrm{cad}=\mathrm{rja} \&$ uact $=8 \& \mathrm{ved}=0 \mathrm{CGMQFjAJ} \& u r l=\mathrm{htt}$ p $\% 3 \mathrm{~A} \% 2 \mathrm{~F} \% 2 \mathrm{Fwww}$.juecesyfiscales.org $\% 2 \mathrm{Fdescargas} \% 2 \mathrm{Ffavorabili}$ dad.zip\%3Fml\%3D5\%26mlt\%3Dsystem\%26tmpl\%3Dcomponent\&ei =OvaqU_75HK_MsQTp64KoDg\&usg=AFQjCNHiLGOc

Hart, H. L. (1963). El Concepto del derecho. Buenos Aires: AbeledoPerrot S.A.

Kawaguchi, H., \& Suárez López, C. (2010). En Suarez, C. Estudios de Derecho penal, p. 379. Bogotá: Universidad Jorge Tadeo Lozano. $\mathrm{R}$ e c u p e r a d o d e http://avalon.utadeo.edu.co/servicios/ebooks/derecho_penal_I/index.h tml

López, D. (2009). ¿Por qué hablar de una "teoría impura de derecho" para América Latina?. En Bonilla , D., D. E. López, et al. Teoría del derecho y trasplantes jurídicos, p. 37-90. Bogotá: Siglo del Hombre Editores.

López Medina, D. E. (2011). El Derecho de los Jueces. Bogotá: Legis. 
Quinche, M. . (2010). Derecho Constitucional Colombiano, de la Carta de 1991 y sus reformas. Bogotá: Doctrina y Ley.

Roxin, C. (2003). Derecho Penal General. Fundamentos. La estructura Teoría del Delito. (Vol. I). Madrid: Civitas.

Siches, L. R. (1974). Direcciones contemporaneas del pensamiento jurídico (la filósofía del Derecho en el siglo XX). Mexico D.F: Editora Nacional.

Uprimny, R., \& Rodríguez, A. (2014). Entre deductivismo y activismo: hacía un intento de recapitulación de los grandes modelos de interpretación jurídica. Recuperado de http://portalweb.u catolica.edu.co/easyWeb2/files/ 116_10314_deductivismoactivismo.pdf

Valenzuela, J. (2014). La teoría del Derecho de H. L. Hart. Recuperado de http://www.juridicas.unam.mx/publica/librev/rev/facdermx/ cont/237/art/art14.pdf

Velasquez, F. V. (2009). Derecho Penal Parte General. Bogotá, Colombia: Librería Jurídica Comlibros. 